\title{
Extending a Teachable Agent with a Social Conversation Module - Effects on Student Experiences and Learning
}

\author{
Agneta Gulz ${ }^{\mathrm{a}}$, Magnus Haake ${ }^{\mathrm{b}}$, Annika Silvervarga \\ ${ }^{\text {a }}$ Department of Computer Science, Linköping University, Sweden \\ ${ }^{\mathrm{b}}$ Department of Design Sciences, Lund University, Sweden
}

\begin{abstract}
The paper discusses the addition of off-task socially oriented conversational abilities to an existing "teachable agent" (TA) in an educational game in mathematics. The purpose of this extension is to affect constructs known to promote learning, such as self-efficacy and engagement as well as enhance students' experiences of interacting with the game. A comparison of students that played the game with the off-task interaction to those who played without it, shows trends that indicate that students who played the game with off-task interaction had a more positive experience of the game, and that they also learnt more, as reflected in the learning outcomes of their TAs.
\end{abstract}

Keywords. Educational game in mathematics, conversational pedagogical agent, teachable agent, off-task interaction, socially oriented conversation.

\section{Introduction}

By conversational pedagogical agents, CPA:s, we refer to computer generated characters in a pedagogical context that engage in spoken or written conversation with students. Some CPAs may use non-verbal conversational channels, such as gestures and facial expressions, but this paper limits itself to conversation in written language. Various research groups have developed CPAs for different domains like physics, mathematics, foreign languages, programming and many others, and several evaluative studies have shown that CPAs can be effective as tutors [e.g. 1, 2]. There is a large variety in how CPA:s are designed and what specific pedagogical strategies they exploit, but all engage, in some way or other, in task-oriented conversation such as: elaborating on students' answers, asking questions regarding the domain or task, correcting misconceptions, asking students to elaborate on their examples, providing hints and directions. In other words, they engage in conversation that clearly pertains to the learning material and tasks in question.

During the past decade some researchers have developed CPAs that in addition to carrying out task-oriented conversation engage in relation oriented or socially oriented conversation with students, i.e. conversation with no (apparent) relation to the learning tasks. Examples are: reassuring or cheering up a student, carrying on smalltalk, engaging in mutual self-disclosure. Relational or social behaviours can also be realized via non-verbal communication, but this is outside the scope of this paper.

Reasons for adding a capability for socially oriented conversation in a CPA include: i) increased overall engagement and receptivity [3] ii) improved recall of the learning 
material through emotional engagement [4] in particular because social experiences activate the reward circuitry of the brain, which helps cement newly learned associations [5], iii) promotion of trust and rapport-building [6] and finally that students may feel more at ease with a learning task or topic [7]. For a more extensive presentation of these and other reasons, see [8, 9]. This paper describes a teachable agent that has been extended with a social conversation module. We discuss the justification for the extension and present an empirical study that evaluates the effects of the extension in terms of student experiences and learning. However, first we present selected examples of other CPAs capable of socially oriented conversation.

\section{Previous Work}

CPAs with a capability for socially oriented conversation broadly belong to two different categories. CPAs in the first category exhibit on-task sociability, that is they will and cannot digress into other topics than those that pertain to the learning task and domain. However, in connection with task-oriented conversation, they exhibit social behaviour such as displaying encouragement, assurance, agreement, and praise. One example is the cooperative co-learner [10] that in addition to on-task conversation in the domain of English language idioms, compliments and shows concern and encouragement when the difficulty level of the questions increase or when the student fails on a question (e.g. "You'll get the next one”). As another example consider the Low social and High Social agents [11] in a system for supporting collaborative design learning regarding thermodynamics. Student pairs can chat with each other as well as with the tutor CPA, where the percentage of social turns by the CPA (showing solidarity with a student who has difficulties, agreeing or showing tension release) is varied from $0 \%$ for the No Social agent, to $15 \%$ for the Low social and to $30 \%$ for the High social. As a third example [12] developed a model of socially intelligent tutorial dialogue on the basis of politeness theory. The polite tutor agent provided tutorial feedback to promote learner face and mitigate face threat, whereas the standard tutor agent provided direct feedback that disregarded learner face.

The second category of socially oriented CPAs contains those that exhibit off-task sociability. These are able to go outside of the task(s) and domain(s) and engage in conversation that involves small-talk-like topics, self-disclosure, personal narratives, etc. Although the work by T. Bickmore does not deal with pedagogical applications per se, it is central in this context. In [6] he coined the term relational agent, an agent designed to develop and maintain long-term, socio-emotional relations with users, and he has conducted a large number of studies that compare relation-oriented and strictly task-oriented agents and explore various off-domain sociability features. The value of autobiographical stories in agents is investigated in [13], with reference to Jakobson's [14] phatic function of dialogue: to keep the communication channel open so that primary functional messages can be conveyed. The authors [13] propose that autobiographical storytelling by an agent is a central means for maintaining user engagement in an intervention over time - which can be crucial for educational applications. Yet they also point at the importance that the stories that an agent tells are truly engaging.

Kumar et al. [2] compared two software versions for letting student pairs engage in collaborative mathematics learning via a chat. Both versions contained cognitive support agents, but one also contained social dialogue agents, designed to show personal 
interest in the students by asking them to reveal their personal preferences about extra-curricular domains. The preferences were used as input when the math problems were constructed, with the intention that the social dialogue should give students the impression that the agent takes personal interest in them. The addition of the social dialogue agents turned out to have a strong positive effect on the attitude that students displayed towards agents and a slight positive effect on learning outcomes.

Mehlman et al. [15] present work on a learning game for expressing conceptual knowledge through qualitative reasoning models. A set of CPAs are included, and among them a quizmaster agent, that besides asking questions and giving feedback makes small talk utterances and humorous distractions unrelated to the quiz domain. This is modelled on how quizmasters in famous television shows countervail participants' stress and provide a more enjoyable form of competition.

\section{A TA Based Game Extended with Social Off-task Conversation}

Our game $[16,17]$ is a mathematics game that trains basic arithmetic skills with a focus on grounding base-ten concepts in spatial representations. It employs a boardgame design with a variety of sub-games. When a student has learnt to play one particular board game, she can teach it to her Teachable Agent (TA) [18]. In the observation mode the TA "watches" the student play and picks up on game rules and on the student's responses to multiple-choice questions, such as "Why did you choose this card?” The student then chooses one answer from the listed potential explanations (but only one correct answer), including a “don't know” option. Proper (or improper) choices of cards and answers promote corresponding skills in the TA throughout the game. In the try-and-be-guided mode, the agent is allowed to propose cards. The student either accepts the agent's suggestion or rejects it and exchanges the agent's card for another one. In the latter case the agent asks, via the multiple-choice-format, why the student thinks her card was a better choice. For more information on the AI in the system we refer to $[16,17]$, which also describe the underlying pedagogical model of a master and an apprentice, which differs from the more common teacher-student model in TA-systems.

In other words, the basic TA-system contains a simple form of on-task conversation, via a multiple-choice format. A simple form of on-task sociability is involved as well, for instance the TA may praise the student when she earns points in the game. ${ }^{1}$ For the study presented here, the game architecture was extended with a module where the student can engage in conversation with the TA, writing freely by means of the keyboard (in contrast to the multiple-choice format in the on-task conversation) and bring up basically any topic in a chat-like manner. We refer to this chat-like conversation as off-task conversation and distinguish within it between on-domain conversation and off-domain conversation - the former referring to chat conversation related to school, math and the math game (but notably not in the sense that the TA provides the student with information to play the games better or understand the math content better), and the latter to any other topics. The off-task conversation is implemented as a mixed-initiative dialogue strategy, which allows both the agent and the

\footnotetext{
${ }^{1}$ Yet a TA, which is merely a student of the learner, cannot coach with respect to whether answers to questions are wrong or right.
} 
user to direct the dialogue by introducing new topics and posing questions. The agent keeps a history of the topics in the dialogue, both the current and previous sessions. On-task and off-task conversation have very different formats, but are still designed as two interrelated and complementary activities. The interconnecting factor is the persona of the agent, which integrates task and domain knowledge with off-domain knowledge (e.g. the agent is a 11-year old that goes to school and is learning math in the game, but also has interests such as music and film).

\subsection{Aims and Relations to other Systems}

The off-task conversation is in the first place a means to enrich the game and its motivational qualities for a novel age group of 12-14 year old users. Informal pre-studies revealed that these users required more variation than younger students who became very engaged by the game in its basic form [19]. Bickmore's work and arguments on how social conversation with agents may be a means to maintain engagement in an intervention over time, was a main source of inspiration. Our aim is accordingly to enhance students' experience and increase their inclination to want to continue to use the game over time. A further aim is to exploit the off-task conversation for pedagogical interventions such as influencing students math self-efficacy and attitudes toward math. It is worth to point out that our work, like the work by others related above, approach off-task conversation in terms of its pedagogical power - not in terms of being pedagogically detrimental in taking attention from the learning task [e.g. 20]. We return to this in the discussion.

Enhancement of students' experience of the game can be achieved in various ways. For some individuals it is a question of variability in order to countervail boredom. For some it is a question of making the learning domain of mathematics more appealing and making students less tense or nervous (cf. Mehlman et al. [15] above). This in turn relates to the potential for more dedicated pedagogical interventions, cf. Kim et al. [7] on affecting students math self-efficacy and detracting "math anxious" students from perceived inabilities to confront mathematical learning material. For such interventions to work, trust in the agent is crucial. Bickmore [6] has shown that small talk and conversational storytelling can contribute to build such trust.

For our system we have taken inspiration from all of the above mentioned research. Nevertheless, our system is unique in involving a Teachable agent capable of offtask, off-domain, sociability. Compared to other pedagogical agents, a TA offers advantages as well as challenges when it comes to developing the agent's off-domain sociability. A TA sits at the very core of an educational software by instantiating the software pedagogy, i.e. learning by teaching. A TA is "someone" who has to learn from the student, and this means that there is an immediate, even if rudimentary, social relation between the student and her TA. Studies have shown that such a social relation develops between students and TAs also on the basis of strict on-task interaction and conversation alone [19, 21]. In other words there is a pedagogically integral and unquestionable sociability of a basic kind to start from and no risk that a pedagogical (teachable) agent is but a misguided social software garniture. While this is an advantage, there is a corresponding challenge in how to develop an adequate off-task conversation that extends and refines the rudimentary sociability of the (same) TA. In an on-task-conversation the relation between student and TA is quite straightforward, with the TA the one who learns and the student the one who teaches. But in an off- 
task-conversation one may for various reasons strive for a more equivalent peerrelation and more mutual learning. For details on how we approach this challenge of designing a peer, while yet retaining some of the protégée-effect [21], see [8].

The present study focused on the potential of the chat to increase engagement by comparing groups of students who used the original game with those who used the extended version, i.e. with and without chat. Apart from students' experiences of the game, we studied their perception of the TA's role, their self-efficacy (i.e. beliefs about their competency in playing the math game), and their learning accomplishments. We also studied possible differences between low- and high-achievers.

\section{Method}

\subsection{Participants and Procedure}

38 female and 42 male 12-14 year olds from three classes in a Swedish school participated in the study. The students were assigned a value (low, middle or high) for math achievement by their teacher, where 18 were classified as low, 39 as middle and 23 as high. Each class was divided into two groups with an even distribution according to gender and math achievement. All students got to play the math game during three lessons. The NoC group used the game without the chat module. The WithC group used the game with the off-task module, and after every two game sessions a "break" was offered. During the first three breaks the students had to chat with the agent until the break ended after three minutes, and the chat was closed. For the breaks thereafter the students were offered a choice between chatting with the agent or continuing to play, and when chatting there was always a choice to end the chat before the break was over. The students in the NoC group groups spent on average a total of 105 minutes with the game and the students in the WithC group 120 minutes, in order to make the time spent on the math game sessions equal for both groups. After the third lesson each student filled out a questionnaire.

It should be pointed out that all students, regardless of condition, did get breaks in the sense of cognitive rest and change of activity. Training one's agent involves an intellectual effort and working on math content, whereas letting one's TA play against the computer only requires passive viewing. For an observer it was obvious that students did made use of the latter as a kind of "break".

\subsection{Instruments and Measurements}

To evaluate the effect of the social chat on learning and experience of the game, a combination of data from questionnaires and computer-generated logs were used. The students filled out a questionnaire with 18 statements scaled from 1 (Strongly disagree) to 7 (Strongly agree). The questionnaire included the areas: i) game experience, e.g. if interesting, challenging, easy to concentrate, ii) experience of the role of the TA in facilitating learning and increasing enjoyment in the game, iii) self-efficacy beliefs regarding the game play and one's role as teacher. Statements for i) and ii) were developed based on [22], the self-efficacy measurement according to guidelines from [23]. Log data was used to see how well each student had taught her agent, as a 
measure of the student's own learning. For the WithC group the logs were also used to gather data regarding students' inclination to chat when given the choice.

\section{Results and Analysis}

Since not all students could participate in all three lessons or fill in the questionnaire, the final analysis included 29 females and 32 males. A comparison of the results on the questionnaire and the knowledge level of the trained agent for the NoC- and WithC-groups is presented in Figure 1. Items were clustered and an average score calculated for the game experience, the perceived importance of the agent's role in the system, and self-efficacy beliefs. The students' learning outcome was calculated based on the agent's final knowledge level in relation to how many times the student had played and trained the agent.

Figure 1 shows that students in the WithC-group tended to have a more positive game experience (diff $=0.54, \mathrm{p}=0.07$ ), but there was no difference in the perceived role of the TA in the game, and marginal differences in self-efficacy beliefs. Also students in the WithC-group tended to reach better result in terms of how well they taught their TA (diff $=0,3, p=0.07)$.
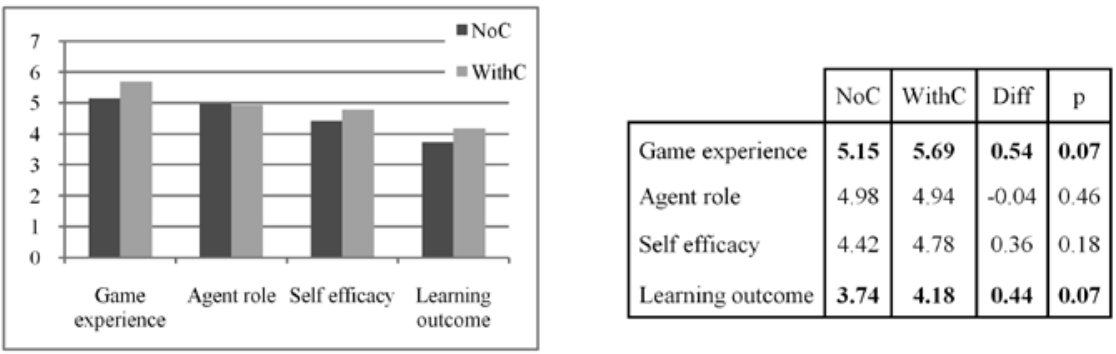

Fig. 1. The table and diagram shows the difference between the NoC- and WithC groups regarding: game experience, the agent's role in the game, self efficacy, and the learning outcome in terms of how well they did train their agent.

Table 1 presents the results separated in sub-groups with respect to students' achievements in mathematics. For the low-achievers we see no differences between the WithC and NoC conditions. However, for the medium and high achieving students the experience of the game is considerably more positive for the WithC condition (diff $=0.71, p=0.04$ and diff $=0.91, p=0.09$ ). High-achievers in the WithC condition also rate their self efficacy beliefs significantly higher ( $\operatorname{diff}=0.93, \mathrm{p}=0.04)$ and have a superior learning outcome (diff=7.65, $\mathrm{p}=0.06$ ).

Table 1. Questionnaire ratings and learning outcomes for low, medium and high achievers.

\begin{tabular}{|l|c|c|c|c|c|c|c|c|c|c|c|c|}
\cline { 2 - 12 } \multicolumn{1}{c|}{} & \multicolumn{4}{c|}{ Low achieving } & \multicolumn{4}{c|}{ Medium achieving } & \multicolumn{4}{c|}{ High achieving } \\
\cline { 2 - 12 } \multicolumn{1}{c|}{} & NoC & WithC & Diff & p & NoC & WithC & Diff & p & NoC & WithC & Diff & p \\
\hline Game experience & 4.92 & 3.58 & -1.35 & 0.12 & $\mathbf{5 . 2 4}$ & $\mathbf{5 . 9 5}$ & $\mathbf{0 . 7 1}$ & $\mathbf{0 . 0 4}$ & $\mathbf{5 . 1 2}$ & $\mathbf{6 . 0 3}$ & $\mathbf{0 . 9 1}$ & $\mathbf{0 . 0 9}$ \\
Agent role & 4.58 & 3.31 & -1.27 & 0.21 & 4.89 & 4.77 & -0.12 & 0.41 & 5.34 & 5.71 & 0.37 & 0.3 \\
Self efficacy & 4.05 & 2.5 & -1.55 & 0.11 & 4.48 & 4.85 & 0.37 & 0.25 & $\mathbf{4 . 5 2}$ & $\mathbf{5 . 4 4}$ & $\mathbf{0 . 9 3}$ & $\mathbf{0 . 0 4}$ \\
Learning outcome & 3.06 & 3.06 & 0.003 & 0.5 & 3.87 & 4.09 & 0.22 & 0.29 & $\mathbf{3 . 9 1}$ & $\mathbf{4 . 6 8}$ & $\mathbf{0 . 7 7}$ & $\mathbf{0 . 0 6}$ \\
\hline
\end{tabular}


For the WithC group we further analysed the chat behaviour for the different subgroups. As shown in Fig. 2, there is a clear pattern where low and medium achievers choose to chat to a much higher extent than high achievers. Comments from students during the lessons indicate that at least some high achievers are quite task oriented and focus at the task at hand, i.e. to teach the agent, and so choose not to chat.
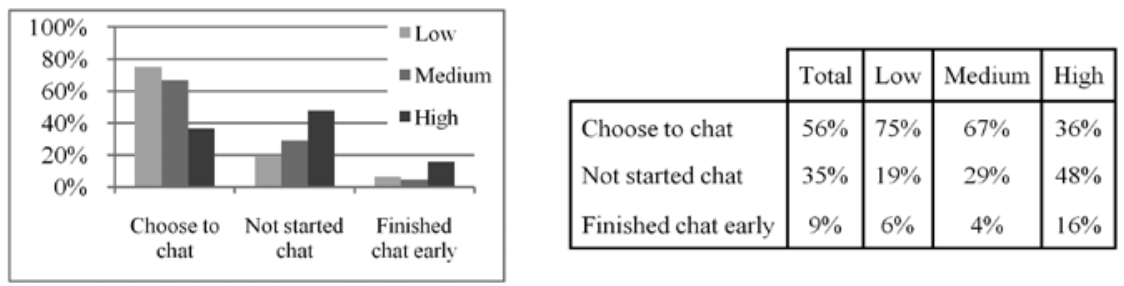

Fig. 2. Table and diagram showing the difference in how low-, medium- and high achievers choose to chat when given the choice.

\section{Discussion}

The primary result of the study is the indication that an added off-task conversation module i) can improve students' game experience and ii) is not necessarily a disadvantage in terms of learning accomplishment, but can to the contrary improve learning. This adds further support to our and others' approaches to the introduction of socially oriented off-task conversation as an integral learning element - in contrast to approaches where off-task behaviour is regarded to divert attention from learning thereby reducing the pedagogical efficiency (e.g. [20]). We hold both kinds of approaches valid, but advocate more nuances in the term "off-task behavior/conversation” in pedagogical contexts, and specifically for digital learning environments. The unit of learning is, we hold, a crucial parameter. For software meant to be used during a set, limited time and in relation to clear learning objectives, it may indeed be relevant to find means to control and even minimize off-task, and also be relatively easy to determine whether a behaviour indeed is unrelated to the curriculum in question. But in relation to a longer term learning context, another kind of balancing must be considered. Off-task behaviour can be essential for the development of a relation between agent and student, which can be central for reaching certain learning goals in longer term. The teachable agent based game discussed in this paper is this kind of longer term learning environment, and it is in view of this that we regard the off-conversation or chat module promising. However, for low achievers - in contrast to middle and high achievers - the chat was not associated with a more positive game experience nor by increased self-efficacy, which prompts further research. One possible, yet speculative, question is whether the addition of linguistic elements and written language to the game is troublesome for low-achievers. This is something that we will need to look further into.

\section{References}

1 Graesser, A., Chipman, P., Haynes, B., Olney, A.: AutoTutor: An intelligent tutoring system with mixed-initiative dialog. In: IEEE Trans. in Education, vol. 48, 612--618, (2005). 
2. Kumar, R., Gweon, G., Joshi, M., Cui, Y., Rose, C.P.: Supporting students working together on math with social dialogue. Proc. the SLaTE Workshop on Speech and Language Technology in Education, pp. 96--99 (2007).

3. Cooper, B., Baynham, M.: Rites of passage: embedding meaningful language, literacy and numeracy skills in skilled trades courses through significant and transforming relationships. National Research and Development Centre for Adult Literacy and Numeracy (2005).

4. Hamann, S.: Cognitive and neural mechanisms of emotional memory. Trends in Cognitive Sciences, 5(9), 394--400 (2001).

5. Chen, J., Shohamy, J., Ross, V., Reeves, B., Wagner, A.: The impact of social belief on the neurophysiology of learning and memory. Society for Neuroscience. San Francisco (2009).

6. Bickmore, T.: Relational Agents: Effecting Change through Human-Computer Relationships. PhD Thesis, Media Arts \& Sciences, Massachusetts Institute of Technology, (2003).

7. Kim, Y., Wei, Q., Xu, B., Ko Y., Ilieva, V.: MathGirls: Increasing girls’ positive attitudes and self-efficacy through pedagogical agents. Proc. AIED 2007, pp. 119-126 (2007).

8. Gulz, A., Haake, M., Silvervarg, A., Sjödén B., Veletsianos, G.: Building a social conversational pedagogical agent - design challenges and methodological approaches. In: D. PerezMarin and I. Pascual-Nieto (eds.) Conversational Agents and Natural Language Interaction: Techniques and Effective Practices, IGI Global (2011).

9. Silvervarg, A., Gulz, A., Sjödén, B.: Design for off-task interaction - Rethinking pedagogy in technology enhanced learning. 10th IEEE Int. Conf. Adv. Learning Technologies, (2010).

10.Maldonado, H., Lee, J., Brave, S., Nass, C., Nakajima, H., Yamada, R., Iwamura, K., Morishima, Y.: We learn better together. In: Proc. CSCL 2005, pp. $408--417$ (2005).

11.Ai, H., Kumar, R., Nguyen, D., Nagasunder, A., Rosé, C.: Exploring the effectiveness of social capabilities and goal alignment in computer supported collaborative learning. In: Proc. ITS 2010. LNCS vol. 6095, pp. 134--143. Springer, Berlin (2010).

12.Wang, N., Johnson, W.L., Mayer, R.E., Rizzo, P., Shaw, E., Collins, H.: The politeness effect. Int. J. Human Computer Studies 66, 96--112 (2008).

13.Bickmore, T., Schulman, D., Yin, L.: Engagement vs. Deceit: Virtual humans with human autobiographies. In: Proc. IVA 2009, pp. 6--19 (2009).

14.Jakobson, R.: Linguistics and Poetics. In: Sebeok, T.A. (ed.) Style in language, pp. 130-144. MIT Press, Cambridge, MA (1960).

15.Mehlmann, G., Häring, M., Bühling, R., Wißner, M., André, E.: Multiple agent roles in an adaptive virtual classroom environment. In: Proc. IVA 2010, pp. 250-256 (2010).

16.Pareto, L., Haake, M., Lindström, P., Sjödén, B., Gulz, A.: A Teachable Agent Based Game Affording Collaboration and Competition (under revision).

17.Pareto, L., Schwartz, D., Svensson, L.: Learning by guiding a teachable agent to play an educational game. In: Proc. AIED 2009, pp. 662--664 (2009).

18.Biswas, G., Katzlberger, T., Bransford J., Schwartz, D.: Extending intelligent learning environments with TA:s to enhance learning. In: Proc. AIED 2001, pp. 389-397 (2001).

19.Lindström, P., Haake, M., Sjödén, B., Gulz, A.: Matching and mismatching between the pedagogical design principles of a math game and the actual practices of play. J. Computer Assisted Learning, 27, 90--102 (2011).

20.Rowe, J., McQuiggan, S., Robison, J., Lester, J.: Off-task behavior in narrative-centered Learning environments. In: Proc. AIED 2009, pp. 99--106 (2009).

21.Chase, C., Chin, D., Oppezzo, M., Schwartz, D.: Teachable agents and the protégé effect. J. Science Education and Technology, 18(4), 334--352 (2009).

22.Anderson L.W., Bourke, S.F.: Assessing affective characteristics in the schools. Lawrence Erlbaum Associates, Mahwah, NJ, 2nd ed. (2000).

23.Bandura, A. Schunk, D.H.: Cultivating competence, self-efficacy, and intrinsic interest through proximal self-motivation. J. Personality and Social Psychology, 41, 586-598 (1981). 